\title{
Digital holographic microscopy in human sperm imaging
}

\author{
Igor Crha • Jana Zakova • Martin Huser • \\ Pavel Ventruba $\cdot$ Eva Lousova $\cdot$ Michal Pohanka
}

Received: 29 March 2011 / Accepted: 13 May 2011 / Published online: 11 June 2011

(C) Springer Science+Business Media, LLC 2011

\begin{abstract}
Purpose The aim of this study was to use digital holographic microscopy (DHM) in human sperm imaging and compare quantitative phase contrast of sperm heads in normozoospermia (NZ) and oligoasthenozoospermia (OAT).

Methods DHM spermatozoa imaging and repeated quantitative phase shift evaluation were used. Five NZ and 5 OAT samples were examined. Semen samples were examined by semen analysis and processed for DHM. Main outcome measures were maximum phase shift value of the sperm heads. Differences of the phase shift and in NZ and OAT samples were statistically tested.

Results In NZ samples median phase shifts were in the range 2.72-3.21 rad and 2.00-2.15 in OAT samples. Differences among individual samples were statistically significant $(p<0.001)$ in both groups. Median phase shift according to sperm count was $2.90 \mathrm{rad}$ in $\mathrm{NZ}$ samples and $2.00 \mathrm{rad}$ in OAT samples. This difference was statistically significant $(p<0.001)$.
\end{abstract}

Capsule Quantitative evaluation of the phase shift by digital holographic microscopy could provide new information on the exact structure and composition of the sperm head.

I. Crha $(\bowtie) \cdot$ J. Zakova $\cdot$ M. Huser $\cdot$ P. Ventruba $\cdot$ E. Lousova Department of Gynecology and Obstetrics, Faculty of Medicine, Masaryk University, and Faculty Hospital,

Obilni trh 11,

60200 Brno, Czech Republic

e-mail: icrha@seznam.cz

M. Pohanka

Department of Functional Diagnostics and Rehabilitation,

St. Anne's Faculty Hospital,

Brno, Czech Republic
Conclusion Quantitative evaluation of the phase shift by DHM could provide new information on the exact structure and composition of the sperm head. At present, this technique is not established for clinical utility.

Keywords Digital holographic microscopy.

Sperm imaging - Spermatozoon · Male infertility .

Chromatin integrity

\section{Introduction}

Digital holographic microscopy (DHM) is a noninvasive imaging method of quantitative phase contrast. Its main advantage over a conventional Zernike phase contrast is the quantification. This feature enables much more sophisticated evaluation of image data. For this reason it is particularly useful in cell biology for visualization and evaluation of living cells that exhibit phase-objects properties. It is considered to be a very promising tool in single-cell analysis [1-3]. Following previous experience from study of structural integrity of neoplastic cells [4], we used the DHM in sperm imaging.

The aim of the study was to use DHM in human sperm imaging and compare quantitative phase contrast of sperm heads in normozoospermia and oligoasthenoteratozoospermia.

\section{Materials and methods}

Digital holographic microscopy

Holographic microscopy is based on the interference of the light passing through the object with the light passing out 
of the object. A microscope built in the Laboratory of Optical Microscopy, Institute of Physical Engineering, Faculty of Mechanical Engineering, Brno University of Technology was used for the DHM observations [5]. The light source is a halogen lamp which could be provided with an interference filter to regulate the temporal coherence of the light. From the source, the beam is divided by a diffraction grating into the reference and the object arm. After passing through the condensers, the beam of light is focused on the object in the object arm and on the reference object in the reference arm. The reference object is usually used to correct for the optical aberrations in the image. Two objectives image the reference object and the object into the common image plane of the microscope where the hologram is formed. The hologram is then magnified by the image objective to meet the holographic condition [6-8]. The magnified image is recorded by the CCD camera. The hologram is numerically processed and the image phase and amplitude are computed. The principles and the scheme of the hologram formation and the image reconstruction have already been described [9].

The hologram processing allows various imaging modes: a/ quantitative phase contrast and its 3D visualization (visualizes the dry mass distribution in the specimen), b/ intensity contrast (similar to bright field light microscopy) and $\mathrm{c} /$ dynamic phase difference-visualizes the changes of the cell dry mass distribution over time [4].

The interferometer system of this DHM is achromatic, and thus suitable for spectrally broad-band extended-source illumination that induces strong optical-sectioning property in its imaging process [5].

Four objective lenses $(\times 10$, two as condensers, two as objectives), digital camera Astropix 1.4 and software developed for the camera and for the image reconstruction were used.

The observation chamber for DHM is specific for the construction of the microscope. Neubauer or Makler chambers are not compatible. The chamber we used consisted of a round coverslip (diameter $22 \mathrm{~mm}$ ) glued with silicon adhesive to a stainless steel annular ring spacer ( $0.8 \mathrm{~mm}$ thick, inner diameter of $15 \mathrm{~mm}$, volume $141 \mathrm{ul}$ ). This formed the bottom part of the observation chamber. The chamber was closed after filling with another coverslip (22 $\mathrm{mm}$ diameter) with a vacuum grease seal.

\section{Samples processing}

Five donor NZ samples and 5 OAT samples of men referred for examination and infertility treatment to our clinic were examined. Informed consent was obtained from all participants. The project was approved by the Brno Faculty Hospital Institutional Review Board and ethics commission.
Participants provided the ejaculates produced via masturbation in polypropylene containers in the hospital after an abstinence period of 3-5 days. Semen analysis was performed according to the guidelines of the World Health Organization [10] and the standards of the department [11]. Sperm counts were evaluated using the Neubauer counting chamber. Centrifugation $(2790 \mathrm{~g}$ for $10 \mathrm{~min}$ ) was used to remove seminal plasma.

The sperm pellet was covered by $1 \mathrm{ml}$ of culture medium (Universal IVF Medium, fy Medi-Cult, Denmark) and the "direct swim-up" method [10] was used to prepare the sample. Sperm concentration and motility was examined. Culture medium was used to adjust sperm concentration to $1.0 \times 106 / \mathrm{ml}$ for better conditions for examination. Samples were placed in sterile polypropylene tubes with screw cap (Nunc CryoTubes ${ }^{\mathrm{TM}}$, Thermo Fisher Scientific, Roskilde, Denmark) and after $1 \mathrm{~h}$ at a stable temperature of $36.0 \mathrm{C}$, transported in a portable incubator (K-systems, Birkerod, Denmark) to DHM.

DHM object chamber was filled with spermatozoa in cultivation medium, reference chamber with the same medium without spermatozoa. The examinations were done without immobilization of the spermatozoa.

Assessment of sperm morphology by DHM was modified with the respect to the microscope construction. First, classic phase-contrast mode was used to find best spermatozoa without obvious defects (oval head, no cytoplasmic droplets, straight midpiece and tail) and with progressive motility. All these examinations were done by the same experienced senior embryologist. Then the imaging mode was changed to quantitative phase contrast and the image of the selected sperm was recorded with the phase shift as the third dimension. The range of the phase shift was from 0 to 4.1 radians (rad), pseudocoloured from dark blue (phase shift $0 \mathrm{rad}$ ) to dark red (phase shift $4.1 \mathrm{rad}$ ). Phase shift of examined spermatozoa was in the range 1.55-4.1 rad. The differences were evaluated at $0.15 \mathrm{rad}$ intervals. Three repeated measurements on the same sample were used to test variability.

Statistical analysis

Differences of the phase shift in NZ/OAT samples were tested using two-way ANOVA. Descriptive statistics such as mean, median, standard deviation (SD) and confidence intervals (CI) were used for summary of phase shift of sperms. Data were deemed to be outliers if they met the following conditions: $<\mathrm{Q} 1-1.5 * \mathrm{IQR}$ or $>\mathrm{Q} 3+1.5 * \mathrm{IQR}$, where Q1 is the first quartile (i.e. the 25th percentile), Q3 is the third quartile (i.e. the 75th percentile) and IQR is the interquartile range (i.e. IQR $=\mathrm{Q} 3-\mathrm{Q} 1$ ). A level of statistical significance $\alpha=0.05$ was used. Analyses, detection of outliers and graphical visualization were performed in statistical 
software SPSS 18.0.2 for Windows and STATISTICA 9.1 for Windows.

\section{Results}

Donors were $24.8 \pm 4.2$ years old, body mass index $22.8 \pm$ 3.1, sperm concentration $81.0 \pm 24.1 \times 10^{6} / \mathrm{ml}$, total sperm number $278.6 \pm 37.7 \times 10^{6}$, progressive movement $51.0 \pm$ $9.6 \%$, normal sperm morphology $52.8 \pm 3.4 \%$. OAT men were $29.5 \pm 5.1$ years old, body mass index $24.2 \pm 4.5$, sperm concentration $4.5 \pm 3.3 \times 10^{6} / \mathrm{ml}$, total sperm number $11.2 \pm$ $7.7 \times 10^{6}$, progressive movement $5.0 \pm 3.6 \%$, normal sperm morphology $7.0 \pm 5.4 \%$.

In one measurement 100 spermatozoa were examined. In the same sample three repeated measurements were done. Eighty seven outliers $(2.9 \%)$ out of the total count of 3000 examined sperms were excluded from the analyses. Differences in the phase shift of the remaining 2913 sperms were tested using two-way ANOVA. As there were statistically significant differences in 4 measured repetitions $(13.3 \%)$ in some samples, two out of three measures of these samples were used for analyses of the phase shift according to the sample and the NZ/OAT group. In NZ group, median phase shifts according to samples were in the range 2.72$3.21 \mathrm{rad}$ and 2.00-2.15 rad in OATgroup. Differences among individual samples were statistically significant $(p<0.001)$ in both groups. Median phase shift according to NZ/OAT group was $2.90 \mathrm{rad}$ in NZ and $2.00 \mathrm{rad}$ in OAT. This difference was statistically significant $(p<0.001)$. Basic descriptive characteristics and parameters are presented in Table 1.
The two-way ANOVA test of the phase shift of sperms according to the sample and NZ/OAT group was performed. Although both parameters were statistically significant, the NZ/OAT group explained more data variability than the sample (sum of squares 422.3 vs. 36.3 , F value 1718.4 vs. 37.0). Hence, the NZ/OAT group is more important parameter rather than the sample.

Images of sperms with different phase shift are presented in Fig. 1.

\section{Discussion}

In this study, DHM demonstrated a significant difference of maximum phase shift of spermatozoa heads in NZ/OAT groups. Differences were found among individual samples, too. All these observations raise the question of the origin of the maximum phase shift. It could be thought that the inner sperm head structure could result in different maximum phase shift. Structural organization of the sperm DNA, condensation of the chromatin, alteration of protamines, histones or other proteins can all be considered $[12,13]$. The relationship between sperm strict morphology and sperm chromatin integrity was examined in some studies [14]. The results suggest that sperm head abnormalities may, in part, be due to incomplete sperm chromatin condensation. These changes could contribute to differences in phase shift of the sperm head.

The sperm chromatine research has brought evidence that supports possible relation between chromosome architecture and optical characteristics of the sperm head. The condensation of DNA into the crystalline-like protamine toroids could infer changes in maximum phase
Table 1 Descriptive statistics of the phase shift according to the sample and NZ/OAT group. Measures which were statistically significantly different from the remained two measures in one sample were excluded from the analysis. ( $\mathrm{NZ}=$ normozoospermia, $\mathrm{OAT}=$ oligoasthenoteratozoospermia)

\begin{tabular}{|c|c|c|c|c|c|c|c|c|c|c|c|c|c|}
\hline \multirow[t]{2}{*}{ Sperm count } & \multirow[t]{2}{*}{ Sample } & \multicolumn{6}{|c|}{ Phase shift (rad) according to samples } & \multicolumn{6}{|c|}{ Phase shift (rad) according to NZ/OAT group } \\
\hline & & Median & Mean & $\mathrm{SD}$ & $95 \%$ & $\mathrm{CI}$ & p-value & Median & Mean & $\mathrm{SD}$ & $95 \%$ & $\mathrm{CI}$ & p-value \\
\hline \multirow[t]{5}{*}{$\mathrm{NZ}$} & A & 2.90 & 2.93 & 0.57 & 2.86 & 2.99 & & & & & & & \multirow[t]{10}{*}{$<0.001^{\mathrm{a}}$} \\
\hline & $\mathrm{B}$ & 3.20 & 3.21 & 0.57 & 3.13 & 3.29 & & & & & & & \\
\hline & $\mathrm{C}$ & 3.13 & 3.00 & 0.63 & 2.91 & 3.09 & $<0.001^{\mathrm{a}}$ & 2.90 & 2.91 & 0.61 & 2.87 & 2.94 & \\
\hline & $\mathrm{D}$ & 2.75 & 2.82 & 0.60 & 2.75 & 2.89 & & & & & & & \\
\hline & $\mathrm{E}$ & 2.75 & 2.72 & 0.59 & 2.65 & 2.79 & & & & & & & \\
\hline \multirow[t]{5}{*}{ OAT } & A & 2.15 & 2.17 & 0.39 & 2.13 & 2.22 & \multirow{5}{*}{$<0.001^{\mathrm{a}}$} & \multirow{5}{*}{2.00} & \multirow{5}{*}{2.10} & \multirow{5}{*}{0.38} & \multirow{5}{*}{2.08} & \multirow{5}{*}{2.13} & \\
\hline & B & 2.15 & 2.23 & 0.42 & 2.18 & 2.28 & & & & & & & \\
\hline & $\mathrm{C}$ & 2.00 & 2.03 & 0.33 & 1.99 & 2.08 & & & & & & & \\
\hline & $\mathrm{D}$ & 2.00 & 2.04 & 0.32 & 2.00 & 2.09 & & & & & & & \\
\hline & $\mathrm{E}$ & 2.00 & 2.00 & 0.33 & 1.96 & 2.04 & & & & & & & \\
\hline
\end{tabular}

\footnotetext{
a Statistically significant difference
} 

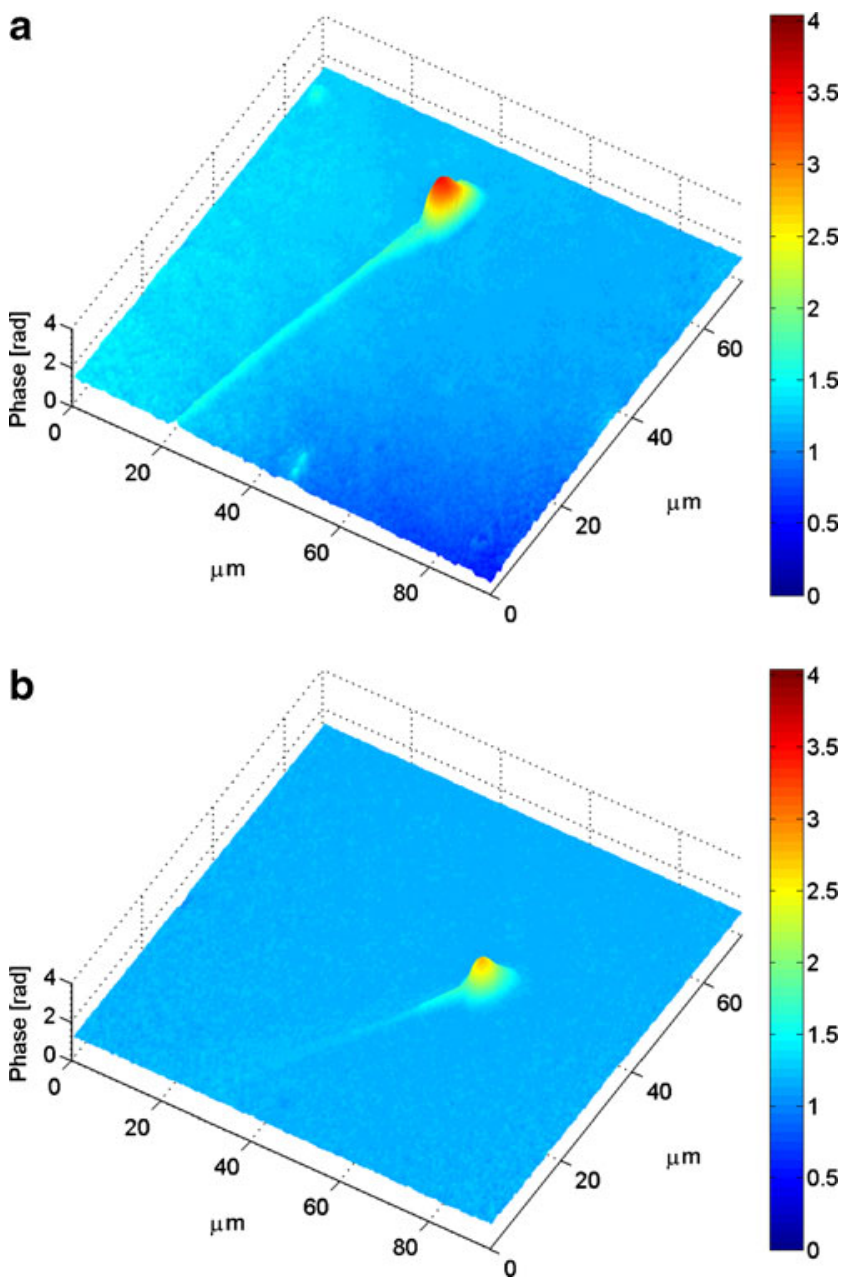

Fig. 1 Digital holographic microscopy images of the sperm with maximal phase shift $3.5 \mathrm{rad}$ (a) and $2.45 \mathrm{rad}(\mathbf{b})$

shift values of the light beam passing through the sperm chromatine $[15,16]$.

Many studies indicate that abnormally low parameters of sperm DNA integrity and sperm membrane integrity correlate and reduce fertility [17-20]. Human sperm head birefringence (double refraction of a ray of light into two rays when it passes through an anisotropic material) was found useful for the selection of spermatozoa for intracytoplasmic sperm injection [21]. Recently, a relationship between the pattern of human sperm head birefringence and DNA fragmentation was described [22]. In-vitro induced nuclear chromatin decondensation of human spermatozoa resulted in significant enlargement of the sperm heads [23]. These observations support the hypothesis that DNA fragmentation could result in differences of the maximum phase shift.

To get more information about the relationship between the phase shift differences and other characteristics of the sperm head, it will be necessary to analyze statistically the results of these examinations (DNA fragmentation, alterations of protamines, histones, strict morphology etc.) in the same sample.

We are aware of the technical limits of this method. Quantitative changes of the phase shift may be affected by the detailed morphology, which was not visible at used resolution (magnification of $40 \times$ followed by a zoom). Exact determination of the maximum phase shift is subjective according to the color spectrum, depending on experience of the staff. There were $2.3 \%$ of outliers and $13.3 \%$ repeated measurements were significantly different in spite of the fact that examinations were done by the same experienced investigator. One of the reasons could be observation of the spermatozoa without immobilization. The "rolling" of the sperm head can interfere with the differences of the repeated measurements. In previous experiments we used distilled water to immobilize the spermatozoa, but this procedure resulted in significant decrease of maximum phase shift. Consequently we prefered study with motile spermatozoa in spite of the fact that the evaluation is more complicated and time consuming.

The maximum value of the phase shift of sperm head depends on the exact location of the sperm head to the optical axis. The phase shift can be affected by different turning of the oval head towards this axis. In the future, the method can be refined by evaluation of the sperm head total phase shift instead of the maximum phase shift. The total phase shift is computed as the sum of the phase shift values in all pixels of the sperm head image and is proportional to the total dry mass of the head. This determination requires higher microscope objectives magnification and more precise resolution between the sperm head and neck. Placement of spermatozoa in a very thin "single layer sperm" will improve stable and exact focusing. Using Makler chamber instead of the current chambers should be optimal. However, this needs specific changes of the microscope construction.

For clinical practice, the time needed for DHM imaging is significant. Sample preparation takes an experienced person approximately $15 \mathrm{~min}$. Total time necessary for measurement of one sample depends on the concentration of measured spermatozoa. In this study, embryologist examined one hundred spermatozoa during 50-90 min. This time should be decreased by higher magnification of microscope objectives and a new chamber with a very thin medium layer.

Of clinical importance is potential contribution of DHM sperm imaging to better IVF results. In previous study we examined twelve OAT semen samples from infertile men from IVF treated couples where four clinical pregnancies were achieved. In some samples we found significantly different values of maximum phase shift of spermatozoa heads. The number of the samples was not sufficient for proper statistical analysis and hence no correlation of higher 
maximum phase shift and pregnancy could be found. Very interesting possibility could be selection and trapping of spermatozoa with high phase shift for ICSI and followup of the embryo development and implantation. Unfortunately, at present it is not possible to combine micromanipulator with DHM.

A great deal of information relevant to the function of the sperm can be obtained from live cells imaging [24, 25]. Research of the sperms by DHM is at the very beginning and many questions remain to be solved. From the fundamental perspective, it must be clarified what structures and substances are involved in the phase shift. It will also be necessary to explain what the relationship is between the quantitative phase contrast values, strict morphology, DNA fragmentation, alterations in protamines and histones in the spermatozoa and infertility.

\section{Conclusions}

Quantitative evaluation of the phase shift by DHM could provide new information on the exact structure and composition of the sperm head that could be useful for clinical practice. At present this technique is not established for clinical utility. Future research requires changes in microscope construction and methods of examination, leading hopefully to better identification of factors responsible for significant changes in the spermatozoon phase shift.

Acknowledgements Authors wish to thank Hana Uhlirova (born Janeckova), M.Sc., Ph.D. from the Institute of Physical Engineering, Faculty of Mechanical Engineering, Brno University of Technology for her kind and skillful technical assistance in DHM.

This work was supported by Internal Grant Agency (IGA) of the Ministry of Health of the Czech Republic-No. NS/9661-4.

\section{References}

1. Marquet P, Rappaz B, Magistretti PJ, Cuche E, Emery Y, Colomb T, et al. Digital holographic microscopy: a noninvasive contrast imaging technique allowing quantitative visualization of living cells with subwavelength axial accuracy. Opt Lett. 2005;30:468-70.

2. Vesely P, Blase C, Matouskova E, Bereiter-Hahn J. Arising podosomal structures are associated with neoplastic cell morphological phenotype induced by the microenvironment. Anticancer Res. 2006;26:967-72.

3. Depeursinge C, Colomb T, Emery Y, Kuhn J, Charriere F, Rappaz B, et al. Digital holographic microscopy applied to life sciences. Conf Proc IEEE Eng Med Biol Soc. 2007;2007:6244-7.

4. Janeckova H, Vesely P, Chmelik R. Proving tumour cells by acute nutritional/energy deprivation as a survival threat: a task for microscopy. Anticancer Res. 2009;29:2339-45.

5. Kolman P, Chmelik R. Coherence-controlled holographic microscope. Opt Express. 2010;18:21990-2003.
6. Chmelik R, Harna Z. Parallel-mode confocal microscope. Opt Eng. 1999;38:1635-9.

7. Chmelik R. Three-dimensional scalar imaging in high-aperture low-coherence interference and holographic microscopes. J Mod Opt. 2006;53:2673-89.

8. Dubois F, Joannes L, Legros JC. Improved three-dimensional imaging with a digital holography microscope with a source of partial spatial coherence. Appl Opt. 1999;38:7085-94.

9. Janeckova H, Kolman P, Vesely P, Chmelik R. Digital holographic microscope with low-spatial and temporal coherence of illumination. In: Optical and Digital Image Processing. Proc. of SPIE 2008; 7000:1-8.

10. World Health Organization Laboratory Manual for the Examination of Human Semen and Sperm-Cervical Mucus Interaction. 4th ed, 1999. Cambridge University Press.

11. Crha I, Kralikova M, Melounova J, Ventruba P, Zakova J, Beharka $\mathrm{R}$, et al. Seminal plasma homocysteine, folate and cobalamin in men with obstructive and non-obstructive azoospermia. J Assist Reprod Genet. 2010;27:533-8.

12. Oliva R. Protamines and male infertility. Hum Reprod Update. 2006;12:417-35.

13. Lefièvre L, Bedu-Addo K, Conner SJ, Machado-Oliveira GS, Chen Y, Kirkman-Brown JC, et al. Counting sperm does not add up any more: time for a new equation? Reproduction. 2007;133:675-84.

14. Zini A, Phillips S, Courchesne A, Boman JM, Baazeem A, Bissonnette F, et al. Sperm head morphology is related to high deoxyribonucleic acid stainability assessed by sperm chromatin structure assay. Fertil Steril. 2009;91:2495-500.

15. Mudrak O, Tomilin N, Zalensky A. Chromosome architecture in the decondensing human sperm nucleus. J Cell Sci. 2005;118:4541-50.

16. Ward WS. Function of sperm chromatin structural elements in fertilization and development. Mol Hum Reprod. 2010;16:30-6.

17. Moskovtsev SI, Willis J, Azad A, Mullen JB. Sperm DNA integrity: correlation with sperm plasma membrane integrity in semen evaluated for male infertility. Arch Androl. 2005;51:33-40.

18. Akgul M, Ozkinay F, Ercal D, Cogulu O, Dogan O, Altay B, et al. Cytogenetic abnormalities in 179 cases with male infertility in Western Region of Turkey: Report and review. J Assist Reprod Genet. 2009;26:119-22.

19. Winkle T, Rosenbusch B, Gagsteiger F, Paiss T, Zoller N. The correlation between male age, sperm quality and sperm DNA fragmentation in 320 men attending a fertility center. J Assist Reprod Genet. 2009;26:41-6.

20. García-Herrero S, Garrido N, Martínez-Conejero JA, Remohí J, Pellicer A, Meseguer M. Ontological evaluation of transcriptional differences between sperm of infertile males and fertile donors using microarray analysis. J Assist Reprod Genet. 2010;27:111-20.

21. Gianaroli L, Magli MC, Ferraretti AP, Crippa A, Lappi M, Capitani $\mathrm{S}$, et al. Birefringence characteristics in sperm heads allow for the selection of reacted spermatozoa for intracytoplasmic sperm injection. Fertil Steril. 2010;93:807-13.

22. Petersen CG, Vagnini LD, Mauri AL, Massaro FC, Cavagna M, Baruffi RL, Oliveira JB, Franco JG Jr. Relationship between DNA damage and sperm head birefringence. Reprod Biomed Online. 2011 Mar 21. [Epub ahead of print] doi:10.1016/j.rbmo.2011.03.017

23. Hammadeh ME, Bernardi A, Zeginiadou T, Amer A, Schmidt W. Relationship between nuclear chromatin decondensation (NCD) in vitro and other sperm parameters and their predictive value on fertilization rate in IVF program. J Assist Reprod Genet. 2005;22:301-5.

24. Kolle S, Reese S, Kummer W. New aspects of gamete transport, fertilization, and embryonic development in the oviduct gained by means of live cell imaging. Theriogenology. 2010;73:786-95.

25. Ierardi V, Niccolini A, Alderighi M, Gazzano A, Martelli F, Solaro R. AFM characterization of rabbit spermatozoa. Microsc Res Tech. 2008;71:529-35. 